\title{
Unsupervised damage detection of CFRP structure with statistical diagnostic method
}

\author{
Atsushi Iwasaki ${ }^{1}$, Akira Todoroki ${ }^{2}$ and Tsuneya Sugiya ${ }^{3}$ \\ 1. Department of Mechanical Engineering, University of Tokyo, Tokyo, Japan \\ 2. Department of Mechanical Engineering and Science, Tokyo Institute of Technology, Tokyo, Japan \\ 3. DMW Corporation, Shizuoka, Japan
}

\begin{abstract}
Structural health monitoring is a noticeable technology for advanced composite structures and civil structures. The present paper proposes a new diagnostic tool for the structural health monitoring that employs a statistical diagnosis of self-learning method. Most of the structural health monitoring systems adopt parametric method based on modeling or non-parametric method such as artificial neural networks. The former method requires modeling of each structure and latter method requires data for the training. And these modeling and data for the training demand much cost. The new statistic diagnosis method does not require the complicated modeling and a learning data of damaged structure for the artificial neural networks.

The present study deals monitoring of delamination of composite beam using change of strain judged by the statistical tools such as Response Surface and F-Statistics. Response surfaces among the measured strain data of surface of composite beam are produced at intact state and monitoring state, and the difference of the each response surface is statistically tested using F-test. As a result, the new method successfully diagnoses the damage without using modeling and a learning data of damaged structure.
\end{abstract}

Key words: Delamination, Damage Diagnosis, Response Surface, F-Statistics, Smart Structure, Unsupervised

\section{INTRODUCTION}

A structural health monitoring system includes numerous mounted sensors, and evaluates the state of an entire structure or structural components in real time. In order to prevent serious failures of civil structures such as bridges and gas piping, structural health monitoring systems have recently become noticeably employed. In Japan especially, minimizing seismic disaster is very important, and development of a system that diagnoses the condition of existing civil structures at low cost is an urgent subject. The present research proposes a new diagnostic method for the structural health monitoring of existing structures at minimal cost. Numerous damage diagnostic methods for structures have been proposed, and most of them employ either a parametric method based on modeling of the structure, or a non-parametric method such as an Artificial Neural Network (ANN). Proposed parametric methods include a substructural flexibility method [1] and a residual force method [2], and proposed non-parametric methods include ANNs [3-5] and response surface methods (RSMs) [6,7]. Parametric methods require modeling of the each structure, and non-parametric methods require numerous data sets for training. Both structural modeling and data sets for training are very costly. In the health monitoring of existing structures, obtaining data sets after creating damage for training of ANN or RSM is almost impossible. This raises significant demand for development of a low-cost diag- nostic method that does not require data on damaged structure.

Therefore, the present study proposes a low-cost statistical diagnostic method for structural damage detection. By a statistical damage detection method, damage is diagnosed from the difference between data obtained in an arbitrary state and data obtained in the present state. Therefore, damage detection requires only data for an arbitrary state, thereby enabling construction of a low-cost damage diagnostic system. Proposed damage detection methods using statistical data processing include damage detection in which change in a time series model is detected[8] and damage detection by use of system identification and similarity test of the identified system (the SI-F method) $[9,10]$.

In the SI-F method, structural damage is detected by statistical tests of similarly of identified systems, which show relations between measured sensor data.

The SI-F method requires only data sets for the non-damaged state, and does not require complicated modeling and numerous data sets after creation of the damage for training ANNs, thereby considerably lowering the cost for creating a diagnostic system.

In the present study, as an example of damage diagnosis by the SI-F method, the present method is applied to delamination detection of a composite beam, and the new damage diagnosis method is investigated experimentally. 


\section{DAMAGE DIAGNOSTIC METHOD}

This section addresses a new damage detecting method using statistical tools. For usual health monitoring systems, relations between measured sensor data and damage location or size are indispensable to identify damages. These relations are derived from modeling of the entire structure or experiments. These works for obtaining the relations are very time consuming and require high computational and/or experimental cost.

The new statistical diagnostic method proposed in the present paper is a low cost simple system. The diagnostic method employs system identification a using response surface(RS) and the damage is automatically diagnosed by testing the similarity of the RS by statistical methods.

The system does not require the relation between measured sensor data and damages. The method does not require a FEM model of the entire structure. This method diagnoses slight change of the relation between the measured sensor data.

\subsection{Damage diagnostic using SI-F method}

Procedure of the new diagnostic method for damage detection is shown in Fig.1 and Fig.2. First, we perform system identification of a structure of intact state using response surface(see below) and create a response surface from the measured sensor data obtained from the initial state(Fig.1). The response surface is named as initial response surface. For example, data of a sensor is selected as a response and the data obtained from the adjacent sensors are selected as predictors. Of course, we can select natural frequencies obtained from vibration data instead of using the measured data directly for the damage detection of entire structure. After the training process, damage monitoring process is started. During the monitoring process(Fig.2), a set of every sensor data is periodically obtained by cycling measurements several times. From the measured set of data, we perform system identification at the structure and a response surface is recreated. The response surface is named as recreated response surface here. The two response surfaces are compared with each other using a statistical similarity test with F-test(see below). When the identified system of monitoring process (=recreated response surface) is discriminated from the identified system of initial state (=initial response surface), it means that relation between the sensor data is changed, and that can be concluded that something happens in the structure. Of course this does not always means damage initiation, but this can provide a low cost solution for damage diagnosis of structure to decide the necessity of the precise investigation.

\subsection{System identification using response surface methodology}

Response surface methodology[6] is used for the system identification in this method. Response surface methodology is employed for the process optimization in a quality engineering field. Response surface methodology consists of a design of experiments to select the most suitable points for fitting the surfaces effectively and the least-square-method to regress response surfaces. Response surface is the approximation function that expresses the relationship between a response and predic-

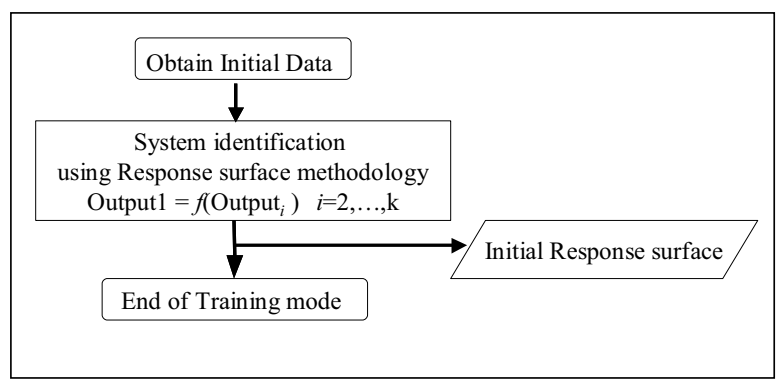

Fig. 1. Flow of training process

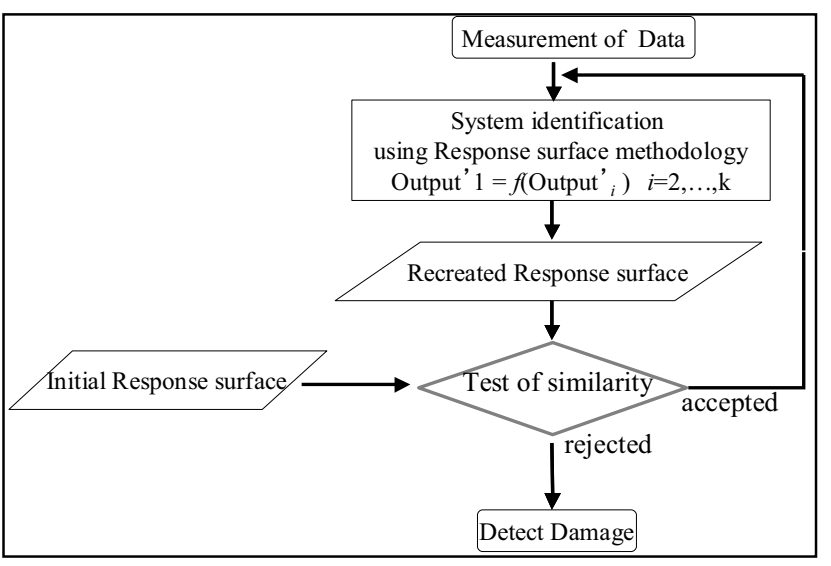

Fig. 2. Flow of monitoring process

tors. Generally, a response surface is represented with the following formula.

$$
y=f\left(x_{1}, x_{2}, \cdots, x_{l}\right)+\varepsilon
$$

Where $x$ 's are predictors, $y$ is a response, $\varepsilon$ is a regression error and $l$ is a number of predictors. In general, polynomials are used.

For simplification, let us consider the case that a response is approximated by quadratic polynomials of two predictors as followings.

$$
y=\beta_{0}+\sum_{j=1}^{l} \beta_{j} x_{j}+\sum_{j=1}^{l} \beta_{j j} x_{j}^{2}+\sum_{i=1}^{l-1} \sum_{j=i+1}^{l} \beta_{i j} x_{i} x_{j}
$$

Where $\beta$ is regression coefficient. If squares or interactions of the predictors $x_{j}^{2}$ and $x_{i} x_{j}$ are replaced by new predictors $x_{j}(j>l)$, the formula (2) becomes the linear regression model as follows.

$$
y=\beta_{0}+\sum_{j=1}^{k} \beta_{j} x_{j}
$$

Where $k$ is number of the predictors after the replacement. In terms of $n$ observations, the Eqn.(3) can be written in matrix form as follows.

$$
\mathbf{Y}=\mathbf{X} \boldsymbol{\beta}+\boldsymbol{\varepsilon}
$$




$$
\mathbf{X}=\left[\begin{array}{ccccc}
1 & x_{11} & x_{12} & \cdots & x_{1 k} \\
1 & x_{21} & x_{22} & \cdots & x_{2 k} \\
\vdots & \vdots & \vdots & \ddots & \vdots \\
1 & x_{n 1} & x_{n 2} & \cdots & x_{n k}
\end{array}\right], \mathbf{Y}=\left\{\begin{array}{c}
\boldsymbol{y}_{1} \\
y_{2} \\
\vdots \\
y_{n}
\end{array}\right\}, \boldsymbol{\beta}=\left\{\begin{array}{c}
\beta_{0} \\
\beta_{1} \\
\vdots \\
\beta_{k}
\end{array}\right\}, \boldsymbol{\varepsilon}=\left\{\begin{array}{c}
\varepsilon_{1} \\
\varepsilon_{2} \\
\vdots \\
\varepsilon_{n}
\end{array}\right\}
$$

Unbiased estimator of $\beta(\mathbf{b})$ is obtained using the least-square-method as follows.

$$
\mathbf{b}=\left(\mathbf{X}^{T} \mathbf{X}\right)^{-1} \mathbf{X}^{T} \mathbf{Y}
$$

\subsection{Similarity test of response surfaces using F-test}

Let us consider, we have two response surfaces that are created from two different sets of experiments.

$$
\begin{aligned}
& \mathbf{Y}_{1}=\mathbf{X}_{1} \boldsymbol{\beta}_{1}+\boldsymbol{\varepsilon}_{1} \\
& \mathbf{Y}_{2}=\mathbf{X}_{2} \boldsymbol{\beta}_{2}+\boldsymbol{\varepsilon}_{2}
\end{aligned}
$$

Where the numbers of experiments for regression are $n_{1}$ and $n_{2}$ respectively. In order to investigate the similarity of the two response surfaces, a null hypothesis is introduced. The hypothetical definition is shown as follows.

$$
H_{0}: \boldsymbol{\beta}_{1}=\boldsymbol{\beta}_{2}
$$

$\Lambda$ ssuming that each error term (c) is independent and has the same distribution in two sets of experiments. In this case, the $\mathrm{F}$-statistic value $F_{0}$ is defined as follows.

$$
\begin{aligned}
& F_{0}=\frac{S S E_{0}-S S E_{12}}{S S E_{12}} * \frac{n-2 p}{p} \\
& n=n_{1}+n_{2} \quad S S E_{12}=S S E_{1}+S S E_{2}
\end{aligned}
$$

Where SSE represents residual sum of squares of a response surface. This F-statistic value $F_{0}$ follows F-distribution of degree of freedom $(p, n-2 p)$ under the null hypothesis. When both of the response surfaces are similar with each other $F_{0}$ becomes small value. Critical limit for the rejection of the hypothesis $H_{0}$ is defined as follows.

$$
F_{0}>F_{p, n-2 p}^{\alpha}
$$

Where $\alpha$ is significance level. The similarity of response surfaces is rejected when $F_{0}$ is larger than $F_{p, n-2}^{\alpha}$.

\section{DAMAGE DETECTION OF CFRP BEAM USING CHANGE OF SURFACE STRAIN DISTRIBUTION}

\subsection{Specimen for experiment}

As mentioned before, the new diagnostic method is applied to delamination monitoring of a CFRP beam, and the effectiveness of the method is experimentally investigated here. Conventional strain gages are employed as sensors in the present study. Specimen configuration of the present study is shown in Fig.3. The specimen is a CFRP cantilever beam with a thickness of $1.4 \mathrm{~mm}$ and stacking sequence of the specimen is $\left[0_{2} / 90_{2}\right]_{\mathrm{S}}$.

Three conventional strain gages are mounted on the specimen surface as shown in the figure. Gage length of center gage is $10 \mathrm{~mm}$ and the gage length of the side gages is $2 \mathrm{~mm}$.

An example of measured data is shown in Fig. 5. Data was measured in $16 \mathrm{KHz}$ for 10 second.

A delamination crack is created under the center gage with a short beam shear test. C-scan image of delamination crack is shown in Fig.4. Schematic illustration of the delamination is shown in Fig.5. Delamination occurs between 00 and 900 plies with matrix cracking in the middle 900ply that is a trigger of the delamination initiation. Length of delamination $(a)$ is defined as Fig.5.

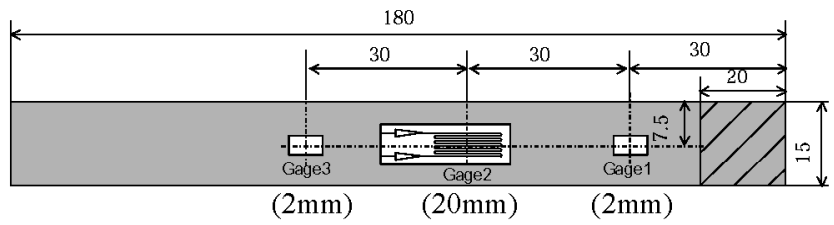

Fig. 3. Specimen configuration

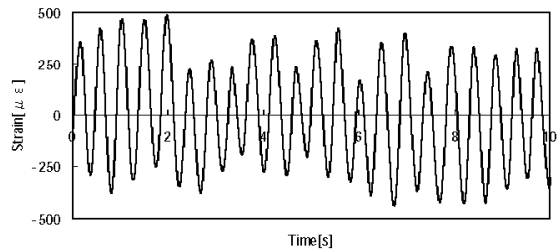

Fig. 4. Strain measurement of Strain gage No.2

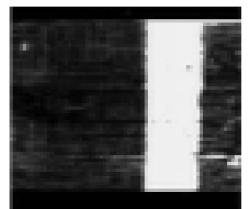

(a)top view

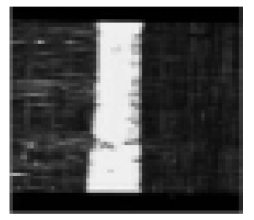

(b) bottom view

Fig. 5. C-Scan images of delamination crack

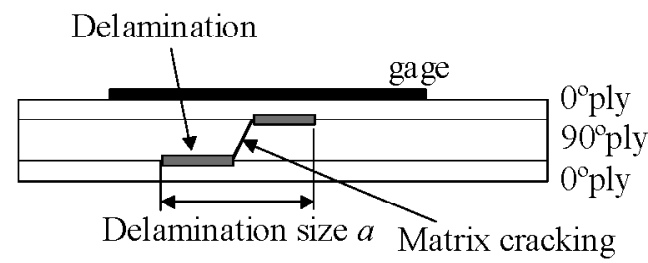

Fig. 6. Schema of delamination 


\subsection{Damage detection method}

In the present study, strain data of the middle gage $\# 2$ is the response, and the strain data of the gage \#1 and \#3 are predictors. Quadratic polynomials are employed for the creation of the response surface. The response surfaces are approximated as follows.

$$
\varepsilon_{2}=\beta_{0}+\beta_{1} \varepsilon_{1}^{2}+\beta_{2} \varepsilon_{3}^{2}+\beta_{3} \varepsilon_{1} \varepsilon_{3}+\beta_{4} \varepsilon_{1}+\beta_{5} \varepsilon_{3}
$$

Where $\varepsilon_{i}$ represents the strain data of gage $\# i$. In the experiments, right end of the specimen is held and excitation is applied to left end of specimen. A response surface of the equation (10) is regressed from the strain 30 data sets selected from time series of strain of gages at random.

The critical limit for the similarity test of response surfaces must first be defined; therefore, similarity tests must be carried out between the response surface of the initial state and the response surface measured before creation of a delamination. In order to obtain distribution of the $\mathrm{F}_{0}$ value of the intact state, 20,000 tests were conducted.

\section{RESULTS AND DISCUSSION}

\subsection{Probability distribution of $\boldsymbol{F}_{\mathbf{0}}$ in intact specimen}

As shown in section 2.3, probability distribution of $F_{0}$ value in a similarity test of same response surfaces follows $\mathrm{F}(p, n-2 p)$. In this case, degrees of freedom $p=6$, total number of response surfaces is 60 , and probability distribution of $F_{0}$ in the intact specimen may follow $\mathrm{F}(6,48)$. Fig. 7 shows experimental values of probability distribution of $F_{0}$ of an intact specimen and $\mathrm{F}(6,48)$ distribution. In the experiment, both the initial response surface and the re-created response surface are created from measured data taken from the intact specimen. The number of trials is 20000 .

As shown in Fig. 7, experimental distribution and theoretical distribution exhibit good agreement. Test of distribution fit is performed as an $\chi^{2}$ test, since $y_{0}$ derived from the following formula follows $\chi^{2}$ distribution.

$$
y_{0}=\sum_{i}^{k} \frac{\left(f_{i}-F_{i}\right)^{2}}{F_{i}}
$$

Where $k$ is number of categories of discrete distribution, $f_{i}$ is sample size contained in each category, and $F_{i}$ is expected size derived from a theoretical distribution. Experimental distribution is divided into 68 categories; therefore, $F_{i}$ must exceed 5.0. The experimental value of $y_{0}$ is 51.5 . Since $5 \%$ critical value $\chi_{69,0.05}^{2}=89.39$, the two distributions are confirmed to fit. Therefore, probability distribution of $F_{0}$ value of the intact structure may follow a distribution that depends on model shape of response surfaces, and critical value of the F-similarity test, which shows the threshold value between the intact state and a damaged state, is derived from only number of data sets and freedom of response surfaces. This result signifies that in the present method threshold value between the intact and damaged states is not affected by noise, variance, and average value of sensor measurement.

In this paper, significance level $\alpha$ for the F-similarity test is set to $5 \%$. In the case where $\mathrm{F}_{6,48}^{0.05}=2.29$ and $\mathrm{F}_{0}$ exceeds this value, similarity of the identified systems is rejected and creation of damage in the structure is diagnosed.

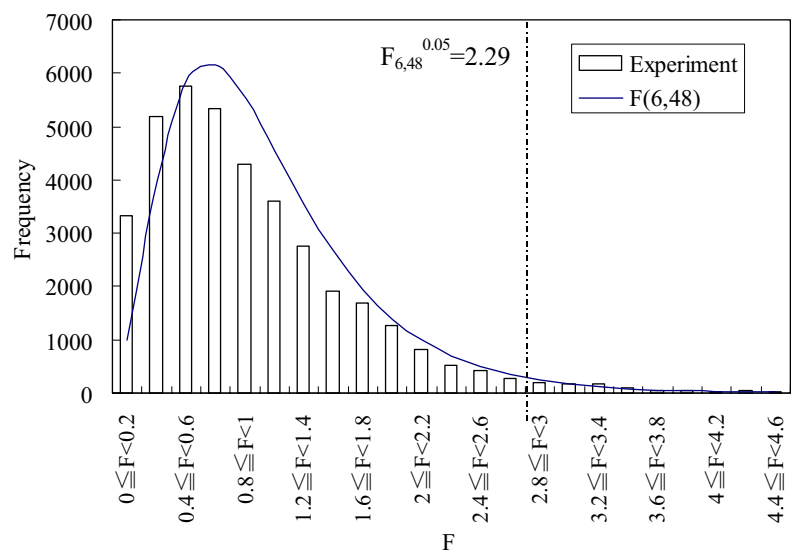

Fig. 7. Frequency distribution of $F_{0}$

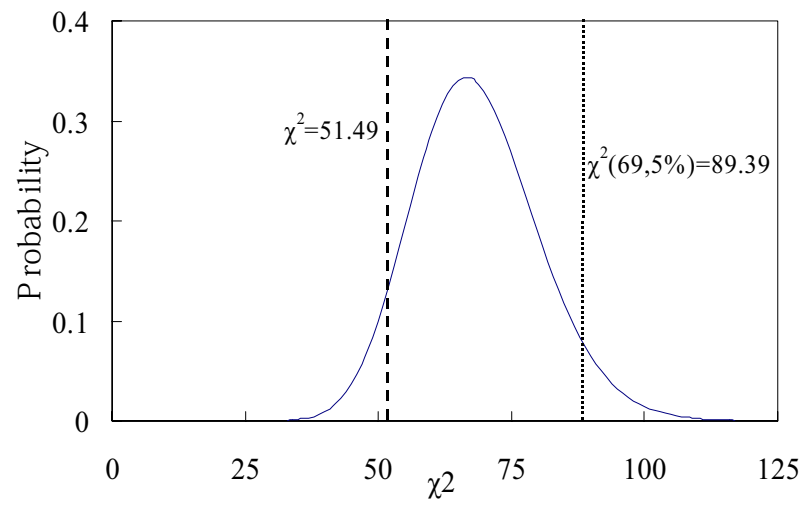

Fig. 8. $\chi^{2}$ distribution

\subsection{Probability distribution of $\boldsymbol{F}_{0}$ in damaged specimen}

After the definition of the limit, delamination detections of the CFRP beams were conducted. 20000 tests of various delamination sizes were conducted. From the experimental difficulties to create delamination cracks of the exact length, the delamination length is quantized into four levels of spacing of $2 \mathrm{~mm}$.

Fig. 9 shows the plot of probability distribution of a damaged structure. Fig. 9(a) shows probability distribution at delamination length $\mathrm{a}=6.1 \mathrm{~mm}$ and (b) shows probability distribution at delamination length $11.3 \mathrm{~mm}$. Each distribution differs from probability distribution of the intact structure shown in Fig.7. This result indicates that $F_{0}$ value is an effective parameter for diagnosing the presence of damage.

Table 1 shows the average value of $F_{0}$ statistics for each size level. $F_{0}$ increases uniformly according to the increase of the delamination length.

Using the limit of 2.29 defined before, the perform- 
ance tests of the diagnostic method are conducted to investigate the effectiveness of the method. Table 2 shows probability of diagnosis. For the in that case (delamination size is zero), the similarity tests of the two response surfaces passed by the performance of $95 \%$. When the delamination crack is longer than $6 \mathrm{~mm}$, the similarity of the two response surfaces is rejected by the performance of $100 \%$, which is perfect diagnosis for delamination existences. On the basis of the performance results, the new diagnostic method provides high performance at low cost. The new method does not require test of damaged state to defined the limit between the intact state and damaged state.

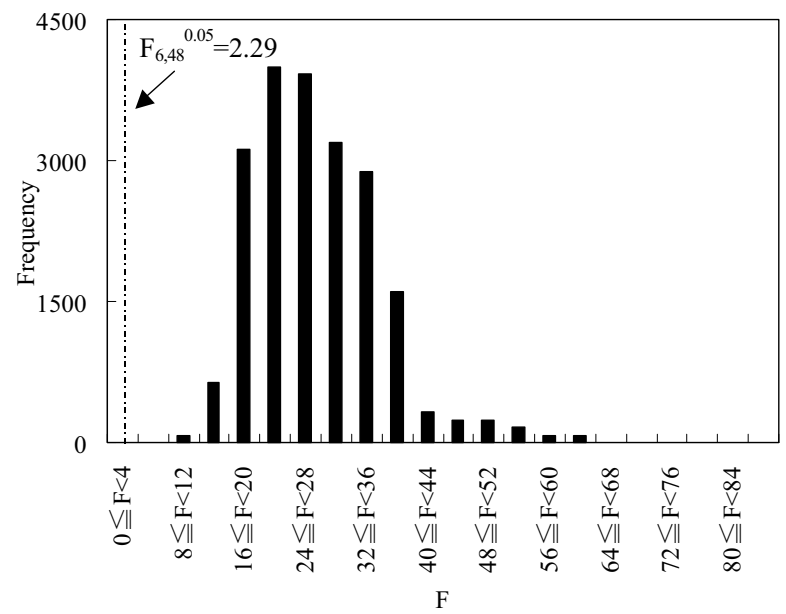

(a) $a=6.1 \mathrm{~mm}$

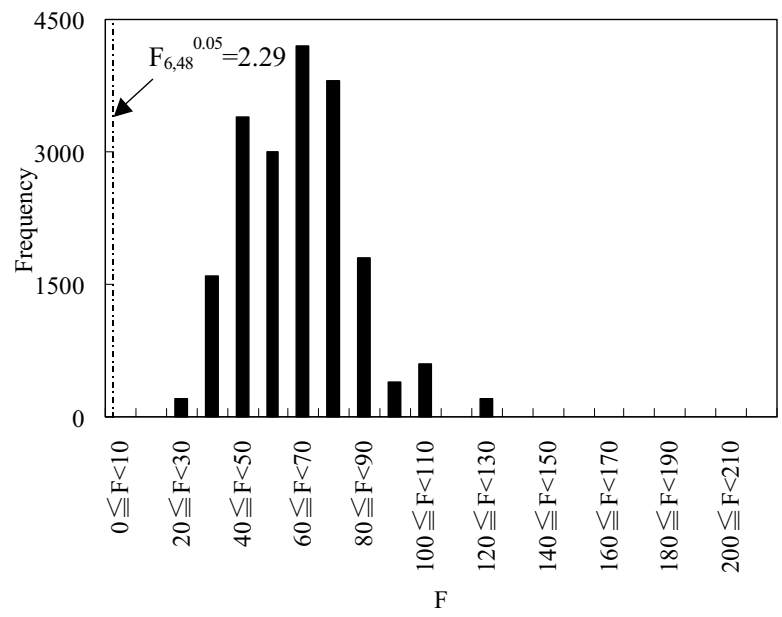

(b) $a=10.8 \mathrm{~mm}$

Fig. 9. Frequency distribution of $\mathrm{F}_{0}$ of damaged specimen
Table 1. Average of $\mathrm{F}_{0}$ of each size region

\begin{tabular}{|c|c|}
\hline Delamination Length & Average of F0 \\
\hline Intact & 1.44 \\
$0<\mathrm{a} \leqq 2$ & 2.46 \\
$2<\mathrm{a} \leqq 4$ & 2.54 \\
$4<\mathrm{a} \leqq 6$ & 2.90 \\
$6<\mathrm{a} \leqq 8$ & 33.18 \\
$8<\mathrm{a} \leqq 10$ & 52.38 \\
$10<\mathrm{a} \leqq 12$ & 59.99 \\
\hline
\end{tabular}

Table 2. Diagnostic accuracy of detection of delamination

\begin{tabular}{|c|c|c|}
\hline Delamination Length & Average of F0 & Reliability of Estimation \\
\hline Intact & 1.44 & $94.8 \%$ \\
$0<\mathrm{a} \leqq 2$ & 2.46 & $62.3 \%$ \\
$2<\mathrm{a} \leqq 4$ & 2.54 & $70.1 \%$ \\
$4<\mathrm{a} \leqq 6$ & 2.90 & $81.3 \%$ \\
$6<\mathrm{a} \leqq 8$ & 33.18 & $100.0 \%$ \\
$8<\mathrm{a} \leqq 10$ & 52.38 & $100.0 \%$ \\
$10<\mathrm{a} \leqq 12$ & 59.99 & $100.0 \%$ \\
\hline
\end{tabular}

\section{CONCLUSIONS}

By conducting similarity tests of the two identified system with F-test, the present paper describes the new damage diagnostic method without measurements of damaged state. As an example of the damage diagnosis by this method, the present study deals monitoring of delamination detections of a composite beam. The delaminations are detected from the slight changes of measured strain data mounted on the specimen surface by using the new statistical tools. The new method employs response surfaces and F-Statistics to discriminate two response surfaces obtained from different sets of measured data, the results obtained are the follows.

(1) A new statistical diagnostic method is proposed. The method discriminates the similarity of the two response surfaces that are created two sets of measured data using the statistical F-test.

(2) Critical limit to discriminate the intact state from the damaged state can be defined only from the $F_{0}$-distribution of the intact state. This causes that the method does not require a large number of experiments of damaged state.

(3) The new method is applied to delamination detections using conventional strain gages. This method successfully detected delamination cracks larger than $6 \mathrm{~mm}$ by the almost perfect performance.

\section{REFERENCES}

1. Felippa C A., Park K C and Justino M R 1998. The construction of free-free flexibility matrices as generalized stiffness inverses Computers \& Structures 68-4 411-418

2. Kameyama M., Ogi Y and Fukunaga H 1999 DAMAGE IDENTIFICATION OF LAMINATED PLATES USING VIBRATION DATA Proc.6th Japan International SAMPE Symposium 987-990

3. Chabg C C, Chang T Y P and Xu Y G 2000, Journal of Intelligent Material Systems and Structures 11 32-42 
4. Kawiecki G and Xu Y G 1999 Journal of Intelligent Material Systems and Structures 10 797-801

5. Zapico J L, Worden K, Molina F J 2001, Smart Materials and Structures 10 553-559

6. Myers R H and Montgomery D C 1995. Response Surface Methodology: Process and Product ptimization Using Designed Experiments John Wiley \& Sons. Inc

7. Todoroki A and Tanaka Y 2002 Delamination identification of cross-ply graphite/epoxy composite beams using electric resistance change method Composite Science and Technology 62-5 629-639

8. Shon H, and Farrar C R 2001 Damage diagnosis using time series analysis of vibration signals Smart Materials and Structure 10 446-452

9. Iwasaki A, Todoroki A, Shimamura Y and Kobayashi H 2001 Statistical Diagnosis for Damage Detection of Self-Learning Smart Structure J of JSME A67-656 771-776 (Japanese)

10. Iwasaki A, Todoroki A, Shimamura Y and Kobayashi H 2002 Unsupervised Structural Damage Diagnostic Method Using Judgement of Change of Response Surface by Statistical Tool (Application for Damage Detection of Composite Structure) J of JSME A68-673 1292-1297 (Japanese) 